\title{
SHOCK MODELLING OF THE BIPOLAR OUTFLOW SOURCE
}

NGC 6537

\author{
L. CUESTA and J. P. PHILLIPS \\ Instituto de Astrofísica de Canarias, E-38200 La Laguna, Tenerife, Spain
}

NGC 6537 is an unusual high excitation bipolar outflow source, with anomalous abundances indicative of a type I nebula. We have recently obtained a range of high resolution spectrophotometry for this source using the $2.5 \mathrm{~m}$ Isaac Newton Telescope (Observatorio del Roque de Los Muchachos, La Palma), together with narrow band optical imaging using the $2.6 \mathrm{~m}$ Nordic Optical Telescope (Observatorio del Roque de los Muchachos, La Palma). As a consequence, it is apparent that the source optical morphology is suggestive of the presence of an hyperbolic shock outflow surface, similar to those observed in other BPN (e.g. NGC 6302, Hb 5). Line ratio maps also indicate the presence of extremely strong [NII] emission at the periphery of the outflow, whilst expansion velocities are of order $\sim 400 \mathrm{~km} \mathrm{sec}^{-1}$ These large shell expansion velocities may in turn be driven by an extremely high velocity wind, which in this case appears to extend over a range $\Delta V \geq 3000 \mathrm{~km}$ $\sec ^{-1}$.

The morphology of this source is highly reminiscent of shock models in which stellar winds directly interact with the ambient medium, leading to shock refraction, appreciable emission in the post-shock zone, and the formation of bi-lobal cavities with typically hour-glass shapes. We have therefore applied such a model to our present results, assuming the ambient medium to vary in density as $R^{-\alpha}$, and the wind to be collimated by a toroidal disk.

The superposition of our model upon optical imaging reveals an extremely close correspondence, whilst a similar superposition of model and spectrophotometric results is also encouraging - with various minor differences attributable to nonsymmetries in the source itself; perhaps a consequence of inhomogeneities within the ambient cloud.

Broadly speaking, we believe that few (if any) such models are capable of so closely replicating the morphology and kinematics of these sources - and in certain cases, it is clear that such structures would also explain the presence of ansae in nebulae such as NGC 3242 and NGC 6905. Similarly, it is clear that whilst care should be taken in the interpretation of our line ratio measures, the high peripheral ratios $[\mathrm{NII}] / \mathrm{H} \alpha$ would be anticipated under a broad variety of shock conditions.

For the present case, we conclude by noting that although a value $\alpha=2$ has been assumed in the models described here, similar (if somewhat poorer) fits may be achieved for the values $\alpha=0$ and 1. Similarly, we determine a source inclination i $=117$ to the line of sight, and require wind velocities $\mathrm{V}_{w} \simeq 900 \mathrm{~km} \mathrm{sec}^{-1}$; although here again, these values are closely similar for all of our model fits. 